\title{
Special issue on green radio
}

\author{
Jacques Palicot ${ }^{1 *}$, Steve McLaughlin ${ }^{2}$, Honggang Zhang ${ }^{3}$ and $\mathrm{H}$ Vincent Poor ${ }^{4}$
}

Society has grown increasingly aware of the need to reduce $\mathrm{CO}_{2}$ emissions in order to mitigate consequential environmental effects. The primary contributors to $\mathrm{CO}_{2}$ emissions are electric power generation, transport, and manufacturing. Information and Communications Technology (ICT) at present contributes a non-negligible $3 \%$ of world-wide energy consumption, which in turn causes about $2 \%$ of the world-wide $\mathrm{CO}_{2}$ emissions. The dramatic growth in Internet traffics and the growing pervasive use of mobile and wireless technologies to support this are likely to increase $\mathrm{CO}_{2}$ emissions in the future unless greater energy efficiency can be achieved in networking technologies. Thus, thedevelopment of wireless networks that require lower energy consumption is desirable. In addition to this key driver of reducing $\mathrm{CO}_{2}$ emissions, the cost of energy has increased substantially and this is a significant proportion of the costs of any wireless access infrastructure company. These issues have resulted in an increased interest in "green radio" networks that reduce the energy requirements for wireless communications, and can thus contribute not only to goals for sustainable development, but also to the profitability of the telecommunication industry.

Although green radio can be justified in the context of energy consumption as noted above, it also can be viewed in a wider sense, such as in the optimization of spectrum usage to reduce electromagnetic radiation levels in order to enable coexistence of multiple wireless systems (i.e., less interference) as well as to reduce human exposure to radiation, in the recycling and reuse of ICT equipment, and in many other related contexts. There are 17 papers in this special issue. It is difficult to classify these papers into distinct categories, as many of them deal with several aspects of green radio. Nevertheless, the classification proposed by Luis Suarez, Loutfi Nuaymi, and Jean-Marie Bonnin in their overview paper (see below) seems very appropriate for classifying these papers and, referring to Figure 1, we can make use of this classification as follows. In particular, this special issue has assembled papers

\footnotetext{
* Correspondence: jacques.palicot@supelec.fr

${ }^{1}$ SUPELEC/IETR Avenue de la Boulais, CS 47601, Cesson-Sévigné Cedex 35576, France

Full list of author information is available at the end of the article
}

dealing with network considerations at large (both indoor and outdoor networks), with the cell layout adaptation (CLA) level (six papers), and with efficient techniques for the radio resource management and optimal transmission (EE-RRM-OT) layer (seven papers). Moreover, three papers deal with cognitive radio networks, belonging to the environment learning \& information exchange (EL-IE) layer, while two others deal specifically with component optimization aspects, pertaining to the component baseline layer, and one provides a tutorial on some green radio topics.

The paper providing an overview of green radio is "An overview and classification of research approaches in green wireless networks" by Luis Suarez, Loutfi Nuaymi, and Jean-Marie Bonnin. It provides an overview of research directions, both at the component level and at the network level. This overview provides an interesting classification, as noted above, and in addition describes major projects dedicated to green radio. It incorporates 76 references, which provide resources for further reading on all aspects of the subject.

Among the six papers dealing with the CLA layer, the paper entitled "Mobile operators have set ambitious targets-is it possible to boost network capacity while reducing its energy consumption?" by Gilbert Micallef, Preben Mogensen, and Hans-Otto Scheck, deals with the challenges faced by telecommunication operators. It quantifies, through a number of case studies, the impact of specific solutions and how the energy consumption trend can be expected to develop over the next decade. It shows that a hybrid macrocell-picocell upgrade is more energy-efficient than a macrocell or picocell only solution. Results show that network operators can get relatively close to their targets, with energy reductions of up to $40 \%$. The next paper, entitled "Evaluation of the potential for energy saving in macrocell and femtocell networks using a heuristic introducing sleep modes in base stations" by Willem Vereecken, Margot Deruyck, Didier Colle, Wout Joseph, Mario Pickavet, Luc Martens, and Piet Demeester, deals with sleep modes for base stations. It derives a heuristic that can serve as a design tool for establishing a baseline. The authors demonstrate 


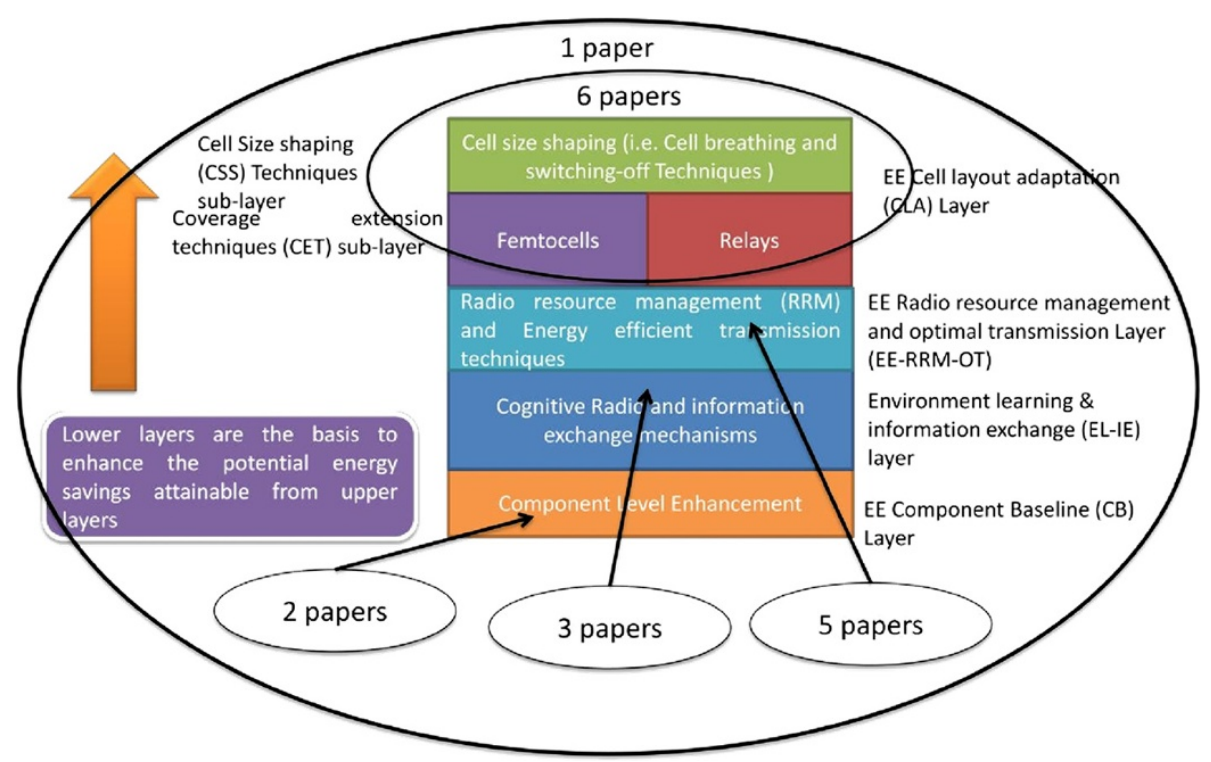

Figure 1 Classification of the 17 papers of the green radio SI based on the classification proposed by Suarez, Nuaymi, and Bonnin in "An overview and classification of research approaches in green wireless networks".

that sleep modes can lead to significant improvements in energy efficiency and act as an enabler for femtocell deployments. The paper "HopScotch-a low-power renewable energy base station network for rural broadband access," by Colin McGuire, Malcolm R Brew, Faisal Darbari, Gregour Bolton, Anthony McMahon, David H Crawford, Stephan Weiss, and Robert W Stewart, describes an example of broadband access to communities in sparsely populated rural areas running in the Scottish Highlands and Islands. Base stations are powered by a combination of renewable sources creating a low cost and scalable solution. The authors argue that the reliance on renewable power (wind turbines) and the intelligent use of frequency bands (WiFi at $5 \mathrm{GHz}$ and UHF "white space") makes this approach an economical green radio technology that can address the problem of rural broadband access. The next paper entitled "Low energy indoor network: deployment optimization" by Siyi Wang, Weisi Guo, and Tim O'Farrell investigates indoor deployment with a minimum of energy to achieve downlink Quality of Service (QoS). It performs an analysis of two access-technologies (LTE femtocell and $\mathrm{WiFi}$ ) in a realistic dynamic multi-user and multi-cell interference network. Thanks to a novel theoretical framework, the paper's results show that optimizing the location of access points both within a building and within individual rooms is critical to minimizing the energy consumption. The paper "Dynamic power control for energy harvesting wireless multimedia sensor networks" by Mohammed-Amine Koulali, Abdellatif Kobbane, Mohammed El Koutbi, Hamidou Tembine, and Jalel Ben-Othman deals with wireless multimedia sensor networks equipped with photovoltaic cells. It proposes a new complete information Markov Decision Process model to characterize a sensor's battery discharge/recharge process and considers the structural properties of optimal transmit policies. Finally in this section the paper entitled "Energy efficiency analysis of one-way and two-way relay systems" by Can Sun and Chenyang Yang provides an in depth and theoretical analysis of energy efficiency for one-way relay transmission and two-way relay transmission and compares these to direct transmission. The paper shows that even relaying, which is often claimed to be a low-energy consumption technique, is not always more energy efficient than direct transmission.

Among the five papers dealing with the RRM and Optimal Transmission Layer, the paper entitled "Resource allocation for energy-efficient cellular systems" by Stefan Videv, John S Thompson, Harald Haas, and Peter M Grant proposes a new scheduler that takes into account energy costs for OFDMA cellular systems. When the energy consumption is dominated by the energy used for data communication as opposed to control channel overhead transmission, a bandwidth expanded mode allows the scheduler to decrease a user's energy consumption by allocating more resource blocks (RBs) and maintaining a constant data rate. The paper further considers a time compression mode, which is a technique that is complementary to bandwidth expanded mode. It allows for energy savings through a reduction of the number of RBs allocated to a user when the energy consumption is dominated by the transmission of signaling traffic. In a realistic framework, the scheduler saves about $38 \%$ of energy compared to a 
frequency selective proportional fair benchmark. The next paper "An energy-efficient opportunistic multicast scheduling based on superposition coding for mixed traffics in wireless networks" by Xing Zhang, Qinzi Li, Yu Huang, and Wenbo Wang discusses with an energy-efficient opportunistic multicast scheduling technique that is based on superposition coding (SC) for multiple mixed unicast and multicast traffic transmission in wireless networks. To improve energy efficiency and system throughput, it considers a combined unicast and multicast SC scheme, which superimposes the unicast or multicast information used by fewer users onto other multicast information used by many users, after dividing all of the traffic into "hot" and "nonhot" traffic based on a certain user number threshold. The paper "Ultra-spectra communication system (USCS): a promising way to energy-efficient green communication networks" by Bin Li, Zheng Zhou, and Weixia Zou proposes a new communication system, named ultra-spectra communication, which combines single cycle modulation with pulse modulation. A performance analysis demonstrates that the overall transmission scheme can indeed be realized within an ultra-low-power framework. The next paper "Energy efficiency in multiaccess fading channels under QoS constraints" by Deli Qiao, Mustafa Cenk Gursoy, and Senem Velipasalar discusses with transmission in a two-user multiaccess fading channels under QoS constraints in the low-power and wideband regimes. Three access types are considered: time-division multiple-access, SC with fixed decoding order, and SC with variable decoding order. The final paper in this grouping, entitled "An energy efficient semi-static power control and link adaptation scheme in UMTS HSDPA" by Yi Huang, Jie Xu, and Ling Qiu, addresses power consumption of the HSDPA system. It proposes a new power control and link adaptation strategy, in which optimal modulation and coding schemes are determined for optimal energy efficiency. This new power control and link adaptation scheme has been extended to the multiple-input multiple-output case and simulation results prove that the gain in energy efficiency is significant and the method is robust.

Among papers dealing with energy-efficient components baseline layer, the paper "Green radio despite 'dirty RF' front-end" by Myriam Ariaudo, Inbar Fijalkow, Jean-Luc Gautier, Mathilde Brandon, Babar Aziz, and Borislav Milevsky deals with the "dirty RF" concept, which refers to the situation in which an imperfect analog front end generates perturbations that can be corrected in the digital domain using signal processing. The authors discuss the implications of this approach for green radio. The paper "Wake-up receiver for radio-on-demand wireless LANs" by Suhua Tang, Hiroyuki Yomo, Yoshihisa Kondo, and Sadao Obana deals with the idle state consumption of wireless local area networks access points. The idea is to wake up the access point only during times of active data communications. It examines the effects of an imperfect radio frequency band pass filter, and suggests soft decision and optimal parameters for both signal detection and signal recognition. The results of the paper confirm that the proposed scheme, for a moderate cost, offers good performance in delivering wake-up signals and controlling false wake-up events caused by wireless local area network signals.

Among the three papers belonging to the EL-IE layer, two papers deal with compressive sensing, which proposed as method for reducing power consumption. Both papers deal with cognitive radio networks. The first of these papers, "A novel reduced power compressive sensing technique for wideband cognitive radio" by Yasin Miar, Claude D'Amours, and Tyseer Aboulnasr, considers sub-Nyquist non-uniform sampling for spectrum sensing to reduce the power consumption of the analog-to-digital converter. The missing samples are estimated by using the expectationmaximization algorithm. The authors show that the proposed sub-Nyquist rate non-uniform sampler is accurate enough to detect the edges of the estimated power spectral density. The second paper, "Efficient blind spectrum sensing for cognitive radio networks based on compressed sensing" by Shancang Li, Xinheng Wang, and Xu Zhou, proposes to improve signal reconstruction algorithms by using $\ell_{\mathrm{p}}$-norm $(0<p<1)$ minimization instead of $\ell_{1}$ or $\ell_{1} / \ell_{2}$ mixed minimization that are commonly used in existing signal recovery schemes. Simulation results show that the proposed solution has a higher spectrum sensing sensitivity and accuracy, and improved reconstruction speed. The third paper dealing with issues at this layer is "Distributed beam forming with phase-only control for green cognitive radio networks" by Xiaohua Lian, Homayoun Nikookar, and Leo P Ligthart. It deals with a new distributed beam-forming technique designed to reach secondary users. By simply controlling only the phase of the transmitted signals, the new method decreases the energy consumption of cognitive radio nodes.

The Guest Editors would like to thank the authors of all papers submitted (both those that were accepted and those that, unfortunately, could not be included) for considering our special issue to disseminate their work. We also would like to warmly thank all the reviewers for their difficult and conscientious work and for the time they spent in reviewing. We also extend our thanks to the JWCN staff, in particular Bernardino McCartney, and to the JWCN Editor-in-Chief Luc Vandendorpe, for offering us the opportunity to present this special issue. We hope that the reader can use the research results presented in these 17 papers to adopt green radio principles in future radio communication systems.

Prof. Jacques Palicot, SUPELEC/IETR, received, in 1983, his PhD degree in Signal Processing from the 
University of Rennes. Since 1988, he has been involved in studies about equalization techniques applied to digital transmissions and analog TV systems. Since 1991, he has been involved mainly in studies concerning the digital communications area and automatic measurements techniques. He has taken an active part in various international bodies EBU, CCIR, URSI, and within RACE, ACTS, and IST European projects. He has published various scientific articles notably on equalization techniques, echo cancellation, hierarchical modulations, and Software Radio techniques. He is currently involved in adaptive Signal Processing and in new techniques as Software Radio, Cognitive radio and Green Radio. From November 2001 to September 2003, he had a temporary position with INRIA/IRISA in Rennes. He serves as Associate Editor for EURASIP JASP since 2008. He also served as lead guest editor for several Special Issues on Software Radio, Cognitive Radio, and Green Radio. He was Technical Program Chairman of CROWNCOM 2009 and Co General Chairman of ISCIT 2011. Since October 2003, he is with Supélec in Rennes where he leads the Signal Communications and Embedded Electronics (SCEE) research team.

Prof. Stephen McLaughlin was born in Clydebank, Scotland in 1960. He received his B.Sc. degree in Electronics and Electrical Engineering from the University of Glasgow in 1981 and the Ph.D. degree from the University of Edinburgh in 1990.

From 1981 to 1984, he was a Development Engineer in industry involved in the design and simulation of integrated thermal imaging and fire control systems. From 1984 to 1986, he worked on the design and development of high-frequency data communication systems. In 1986, he joined the Department of Electronics and Electrical Engineering at the University of Edinburgh as a research fellow where he studied the performance of linear adaptive algorithms in high noise and non-stationary environments. In 1988, he joined the academic staff at Edinburgh, and from 1991 until 2001 he held a Royal Society University Research Fellowship to study nonlinear signal processing techniques. In 2002, he was awarded a personal Chair in Electronic Communication Systems at the University of Edinburgh. In October 2011, he joined HeriotWatt University as a Professor of Signal Processing and Head of the School of Engineering and Physical Sciences. His research interests lie in the fields of adaptive signal processing and nonlinear dynamical systems theory and their applications to biomedical, energy, and communication systems. Prof McLaughlin is a Fellow of the Royal Academy of Engineering, of the Royal Society of Edinburgh, of the Institute of Engineering and Technology and of the IEEE.
Prof. H. Vincent Poor (Ph.D., Princeton 1977) is the Dean of Engineering and Applied Science at Princeton University, where he is also the Michael Henry Strater University Professor. From 1977 until he joined the Princeton faculty in 1990, he was a faculty member at the University of Illinois at Urbana-Champaign. He has also held visiting appointments at a number of universities, including Imperial College, Stanford, and Harvard. His research interests are primarily in the areas of statistical signal processing, stochastic analysis, and information theory, with applications in wireless networks and related fields such as social networks and smart grid. Among his publications in these fields are the recent books Classical, Semi-classical and Quantum Noise (Springer, 2012), and Smart Grid Communications and Networking (Cambridge University Press, 2012). Dr. Poor is a member of the U.S. National Academy of Engineering and the U.S. National Academy of Sciences, a Fellow of the IEEE and the American Academy of Arts \& Sciences, and an International Fellow of the Royal Academy of Engineering of the U.K. He has served as the President of the IEEE Information Theory Society and as Editor-in-Chief of the IEEE Transactions on Information Theory. In 2002, he received a Guggenheim Fellowship, and in 2005 he received the IEEE Education Medal. Recent recognition of his work includes the 2010 IET Ambrose Fleming Medal for Achievement in Communications, the 2011 IEEE Eric E. Sumner Award, a Royal Academy (U.K.) Distinguished Visiting Fellowship (2012), and honorary doctorates from Aalborg University (2012), the Hong Kong University of Science and Technology (2012), and the University of Edinburgh (2011).

Prof. Honggang Zhang is the International Chair Professor of Excellence, CominLabs Excellence Center (Laboratoire d'Excellence), Université Européenne de Bretagne (UEB - European University of Brittany) and Supelec/IETR, France, as well as a Professor of the Department of Information Science and Electronic Engineering, Zhejiang University, China. He is an Honorary Visiting Professor of the University of York, UK. $\mathrm{He}$ received the Ph.D. degree in Electrical Engineering from Kagoshima University, Japan, in March 1999. From October 1999 to March 2002, he was with the Telecommunications Advancement Organization (TAO) of Japan, as a TAO Research Fellow. From April 2002 to November 2002, he joined the TOYOTA IT Centre. From December 2002 to August 2004, he has been with the UWB Research Consortium, Communications Research Laboratory (CRL) and National Institute of Information and Communications Technology (NICT) of Japan. He was the principle author and contributor for proposing DS-UWB in IEEE 802.15 WPAN 
standardization task group. From September 2004 to February 2008, he has been with CREATE-NET (Italy), where he leaded its wireless teams in exploring Cognitive Radio and UWB technologies while participated the European FP6/FP7 projects (EUWB, PULSERS2). Dr. Zhang has served as the Chair of the Technical Committee on Cognitive Networks (TCCN) of the IEEE Communications Society (ComSoc) in 20112012. He was the Co-Chairs of IEEE Globecom 2008 Symposium. He was the founding TPC Co-Chairs of CrownCom 2006 as well as the Steering Committee Member of CrownCom 2006-2009. In the area of cognitive green communications and computing, Dr. Zhang was the Lead Guest Editor of the IEEE Communications Magazine special issues on "Green Communications". He was the General Chair of IEEE/ACM GreenCom 2010 (2010 IEEE/ACM International Conference on Green Computing and Communications) and the Co-Chairs of the IEEE International Workshop on Green Communications (GreenComm 2010-2011) in conjunction with IEEE ICC/Globecom. He is the co-author/editor of two books with the titles of "Cognitive CommunicationsDistributed Artificial Intelligence (DAI), Regulatory Policy \& Economics, Implementation" (John Wiley \& Sons) and "Green Communications: Theoretical Fundamentals, Algorithms and Applications" (CRC Press).

\section{Author details}

1SUPELEC/IETR Avenue de la Boulais, CS 47601, Cesson-Sévigné Cedex 35576 ,

France. ${ }^{2}$ School of Engineering and Physical Sciences, Heriot-Watt University, Edinburgh, Scotland, UK. ${ }^{3}$ Université Européenne de Bretagne- SUPELEC Avenue de la Boulais, CS 47601, Cesson-Sévigné Cedex 35576, France. ${ }^{4}$ School of Engineering and Applied Science Princeton University, C-230 Engineering Quadrangle, Olden Street, Princeton, NJ 08544, USA.

Received: 18 December 2012 Accepted: 18 December 2012 Published: 30 January 2013

doi:10.1186/1687-1499-2013-21

Cite this article as: Palicot et al:: Special issue on green radio. EURASIP Journal on Wireless Communications and Networking 2013 2013:21.
Submit your manuscript to a SpringerOpen ${ }^{\circ}$ journal and benefit from:

- Convenient online submission

- Rigorous peer review

- Immediate publication on acceptance

- Open access: articles freely available online

- High visibility within the field

- Retaining the copyright to your article

Submit your next manuscript at $>$ springeropen.com 